\title{
LncRNA ODIR1 inhibits osteogenic differentiation of hUC-MSCs through the FBXO25/H2BK120ub/ H3K4me3/OSX axis
}

Shiwei He ${ }^{1,2,3}$, Sheng Yang ${ }^{4}$, Yanru Zhang ${ }^{1}$, Xiaoling Li', Dan Gao', Yancheng Zhong ${ }^{1}$, Lihua Cao', Haotian Ma', Ying Liu', Guiyuan Li ${ }^{1}$, Shuping Peng ${ }^{1,2,3}$ and Cijun Shuai ${ }^{5,6}$

\begin{abstract}
Long noncoding RNAs (IncRNAs) have been demonstrated to be important regulators during the osteogenic differentiation of mesenchymal stem cells (MSCs). We analyzed the IncRNA expression profile during osteogenic differentiation of human umbilical cord-derived mesenchymal stem cells (hUC-MSCs) and identified a significantly downregulated IncRNA RP11-527N22.2, named osteogenic differentiation inhibitory IncRNA 1, ODIR1. In hUC-MSCs, ODIR1 knockdown significantly promoted osteogenic differentiation, whereas overexpression inhibited osteogenic differentiation in vitro and in vivo. Mechanistically, ODIR1 interacts with F-box protein 25 (FBXO25) and facilitates the proteasome-dependent degradation of FBXO25 by recruiting Cullin 3 (CUL3). FBXO25 increases the monoubiquitination of H2BK120 (H2BK120ub) which subsequently promotes the trimethylation of H3K4 (H3K4me3). Both $\mathrm{H} 2 \mathrm{BK} 12 \mathrm{Oub}$ and H3K4me3 form a loose chromatin structure, inducing the transcription of the key transcription factor osterix (OSX) and increasing the expression of the downstream osteoblast markers, osteocalcin (OCN), osteopontin (OPN), and alkaline phosphatase (ALP). In summary, ODIR1 acts as a key negative regulator during the osteogenic differentiation of hUC-MSCs through the FBXO25/H2BK120ub/H3K4me3/OSX axis, which may provide a novel understanding of IncRNAs that regulate the osteogenesis of MSCs and a potential therapeutic strategy for the regeneration of bone defects.
\end{abstract}

\section{Introduction}

Tissue regeneration and repair through the differentiation of mesenchymal stem cells (MSCs) has been a hot topic in regenerative medicine $\mathrm{e}^{1-3}$. Moreover, the combination of biodegradable composites with mesenchymal stem cells is expected to facilitate their use in tissue and organ repair ${ }^{4-7}$. MSCs have strong proliferative ability and

Correspondence: Shuping Peng (shuping@csu.edu.cn) or

Cijun Shuai (shuai@csu.edu.cn)

${ }^{1} \mathrm{NHC}$ Key Laboratory of Carcinogenesis, Hunan Provincial Tumor Hospital,

Central South University, Changsha 410013, China

${ }^{2}$ The Key Laboratory of Carcinogenesis and Cancer Invasion of the Chinese

Ministry of Education, Cancer Research Institute and School of Basic Medical

Sciences, Central South University, Changsha 410078, China

Full list of author information is available at the end of the article.

These authors contributed equally: Shiwei He, Sheng Yang

Edited by $\mathrm{G}$. Raschellà multi-lineage differentiation, such as differentiation into fat, muscle, bone, cartilage, and other types of cells ${ }^{8,9}$. In particular, MSCs derived from umbilical cord (hUCMSCs) are expected to be a new potential seed cell in tissue engineering ${ }^{9,10}$. The hUC-MSCs can differentiate into osteoblasts and may serve as a potential cell source for bone tissue engineering ${ }^{11,12}$. After hUC-MSCs incubated with osteogenic differentiation medium, the expression levels of the crucial differentiation transcription factors runt-related transcription factor-2 (RUNX2), osterix (OSX) and osteocalcin (OCN) were increased ${ }^{13}$.

Long non-coding RNAs (lncRNAs) are a class of RNA molecules longer than 200 nucleotides that unable to encode proteins. In recent years, increasing numbers of studies have shown that lncRNAs are involved in the osteogenesis of MSCs by regulating osteogenic

\section{(c) The Author(s) 2019}

(c) (i) Open Access This article is licensed under a Creative Commons Attribution 4.0 International License, which permits use, sharing, adaptation, distribution and reproduction cc) in any medium or format, as long as you give appropriate credit to the original author(s) and the source, provide a link to the Creative Commons license, and indicate if changes were made. The images or other third party material in this article are included in the article's Creative Commons license, unless indicated otherwise in a credit line to the material. If material is not included in the article's Creative Commons license and your intended use is not permitted by statutory regulation or exceeds the permitted use, you will need to obtain permission directly from the copyright holder. To view a copy of this license, visit http://creativecommons.org/licenses/by/4.0/. 
transcription factors, such as RUNX2, DLX5, and OSX ${ }^{14}$. LncRNA ANCER inhibited the osteogenic differentiation of periodontal ligament stem cells (hPLSCs) via blocking the canonical WNT signaling pathway ${ }^{15}$, while lncRNA KCNQ1OT1 promoted osteogenesis through activating WNT signaling pathway ${ }^{16}$. LncRNA MEG3 promoted the osteogenic differentiation of MSCs by mediating BMP4 transcription activation ${ }^{17}$, and inhibited osteogenesis of BMSCs by suppressing miR-133a-3p ${ }^{18}$. LncRNA PRNCR1 increased the expression of CXCR4 through inhibiting miR-211-5p, then inhibited osteogenic differentiation and resulted in osteolysis after hip replacement ${ }^{19}$. Interestingly, during the human adipose-derived stem cells (hADSCs) osteogenic differentiation process, lncRNA MIR31HG promoted phosphorylation of I $\mathrm{KB} \alpha$ via directly binding to IкB $\alpha$ and NF- $\kappa B$, while the nuclear translocation of NF- $\mathrm{kB}$ combined to MIR31HG promoter and prompted its expression ${ }^{20}$. However, the roles and regulatory mechanisms of lncRNAs during hUC-MSCs osteogenic differentiation require further exploration.

In this study, we conducted a high-throughput Agilent Human lncRNA Microarray during hUC-MSCs differentiation. The lncRNA RP11-527N22.2 was significantly decreased during osteogenic differentiation of hUC-MSCs and was named osteogenic differentiation inhibitory regulator 1 (ODIR1). The biological functions and mechanism of ODIR1 in hUC-MSCs has not been reported previously. Therefore, our study demonstrates a novel ODIR1/FBXO25/OSX regulatory network regulating the osteogenic differentiation of hUC-MSCs, which may provide a potential strategy to induce osteogenic differentiation for bone regeneration.

\section{Results}

\section{ODIR1 is downregulated during hUC-MSCs osteogenic} differentiation

The hUC-MSCs cells were derived from human umbilical cord and its differentiation potential has been researched previously ${ }^{11,12}$. QC1205 is a hUC-MSCs cell line and is used in this study. The specific surface markers of the QC1205 cell line were characterized by flow cytometry, including CD44-FITC (99.7\%), CD73-PE (99.8\%), CD90-PerCP (99.7\%), CD29-PE (99.9\%), and CD34-APC (0.102\%) (Supplemental Fig. 1). The expression of OSX, RUNX2 and alkaline phosphatase (ALP) were used to explore the optimum DXM concentration in osteogenic differentiation medium (OM) for hUC-MSCs, and the data showed that the optimum concentration of DXM was $100 \mathrm{nM}$ (Supplemental Fig. 2A, B).

To verify their osteogenic differentiation ability, QC1205 cells were incubated with proliferation medium (PM) or osteogenic medium (OM) for 7, 14, or 21 days, respectively. After 21 days, the morphology and cytoskeleton (F-actin staining) of the differentiation group were significantly altered, and the cell size and the nucleus became larger than those in control group (Fig. 1a,). The calcium-rich deposits and ALP activity was enhanced in 7 , 14, and 21 days after osteogenic differentiation (Fig. 1b, c). Calcium nodules were analyzed by Scanning Electron Microscopy and Energy Dispersive Spectroscopy, and data suggested that calcium $(\mathrm{Ca})$ and phosphorus $(\mathrm{P})$ elements were increased after 28 days of osteogenic differentiation (Supplemental Fig. 3). These data confirmed that hUCMSCs shown the potency to differentiate into osteoblasts.

To identify specific lncRNAs during osteogenic differentiation, we conducted a lncRNA profile using Agilent Human lncRNA Microarray (OE Biotech Corporation, Shanghai, China) for undiff and diff hUC-MSCs (Fig. 1d left side). The heatmap and table showed that 23 lncRNAs were significantly differentially expressed upon osteogenic differentiation (Fig. 1d and Table S1). We chose a significantly downregulated lncRNA that has not been reported previously, RP11-527N22.2, and named it lncRNA ODIR1. The RNA expression of ODIR1 was reduced in differentiated hUC-MSCs at distinct times ( 7 , 14, and 21 days) (Fig. 1e). Moreover, the expression of the stem cells marker CD44 was significantly decreased, while the critical osteogenic transcription factors RUNX2 and OSX, and the osteogenic marker osteopontin (OPN) were obviously increased (Fig. 1e). These results demonstrated that lncRNA ODIR1 may be related to the osteogenic differentiation of MSCs.

Bioinformatic analysis indicated that ODIR1 was located on chromosome 8 and contains two exons (Supplemental Fig. 4A), with a total full length of $578 \mathrm{bp}$. ODIR1 is mainly located at the nucleus of hUC-MSCs as demonstrated by nucleoplasm separation and FISH assays (Supplemental Fig. 4B, C). In addition, the potential coding ability of ODIR1 sequences was predicted by the Coding Potential Calculator (CPC) program and the coding potential score of ODIR1 cDNA was -1.15585 , which indicated that it did not have protein coding ability (Supplemental Fig. 4D). In addition, we cloned and inserted full-length ODIR1 cDNA with a HA added to its 3' terminus (HA-ODIR1), and transfected 293 cells, with HA-GAPDH as a positive control. RT-qPCR analysis revealed that both the HA-ODIR1 and HA-GAPDH vector were expressed at high RNA levels in 293 cells, while no protein band was detected in the HA-ODIR1 group by western blotting (Supplemental Fig. 4E). Collectively, ODIR1 mainly located on nucleus and was confirmed as a non-coding RNA.

\section{ODIR1 inhibits the osteogenic differentiation of hUC-MSCs in vitro and in vivo}

To investigate the role of ODIR1 in osteogenic differentiation, ODIR1 knockdown or ectopic expression cell lines were established by a lentiviral system. Then stable 


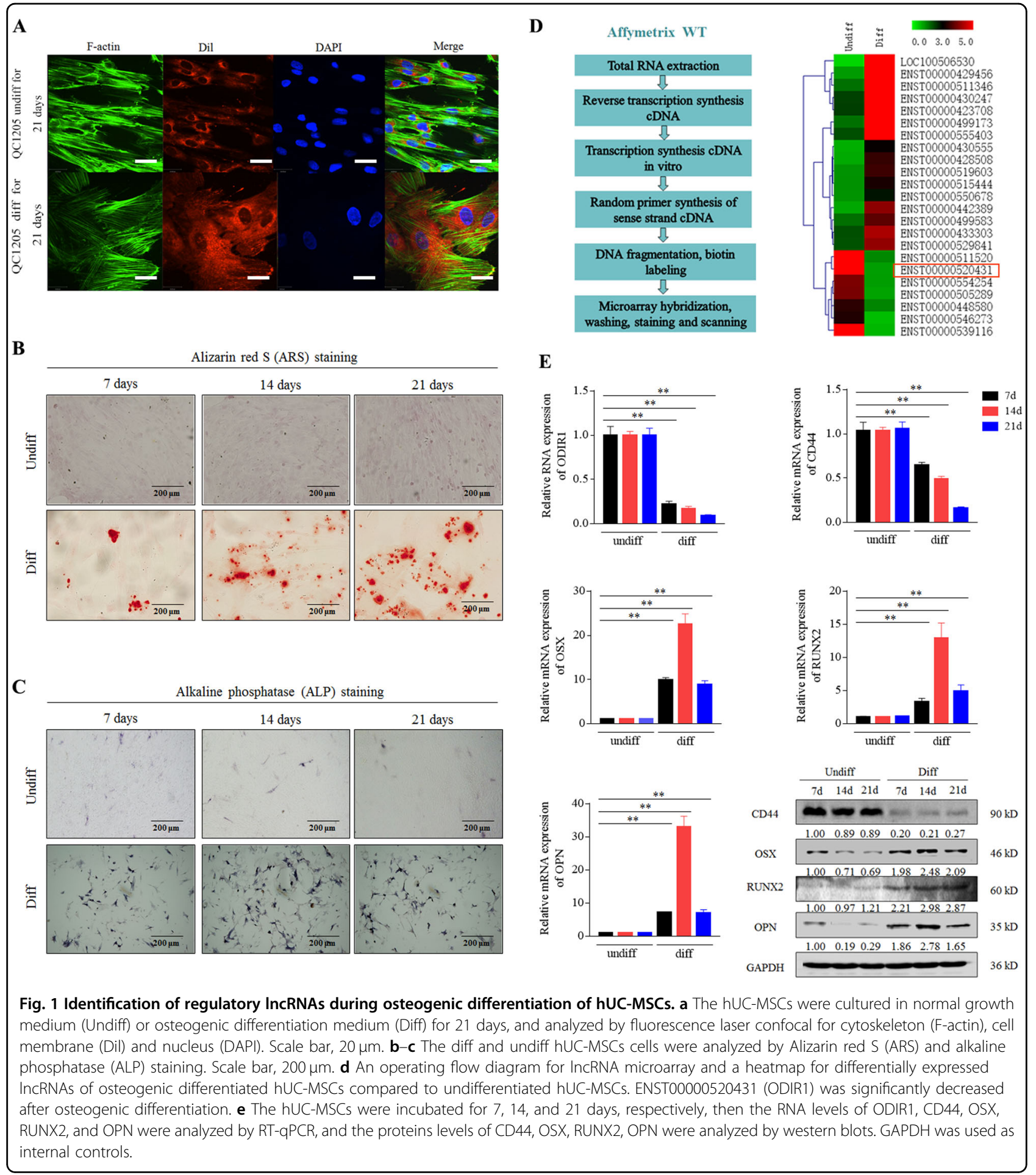

hUC-MSCs were incubated with OM for 21 days and stained using ARS or ALP kit. The results showed that the calcium nodules and ALP activity of the ODIR1 knockdown group were significantly increased (Fig. 2a).

To further verify the role of ODIR1 in the osteogenic differentiation of hUC-MSCs in vivo, a bone formation assay in nude mice was performed using Bio-Oss collagen scaffolds. After 8 weeks of osteogenic differentiation in vivo, all nude mice were sacrificed, and micro-CT analysis data suggested that less new bone formed in the ODIR1 group mice compared with the vector group (Fig. 2b). In addition, BV/TV (bone 

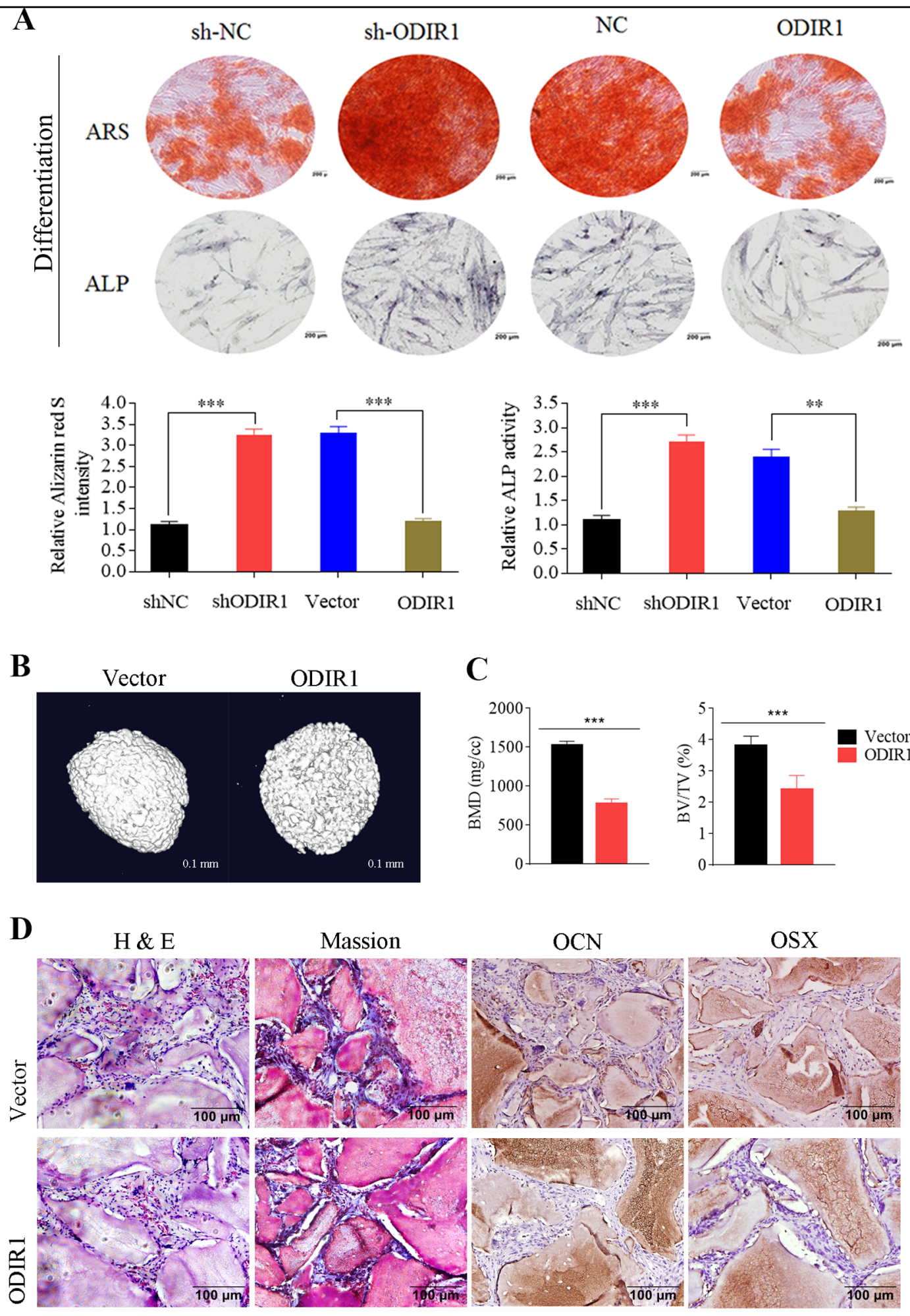

Fig. 2 ODIR1 inhibits the osteogenic differentiation of hUC-MSCs in vitro and in vivo. a The hUC-MSCs were transfected with shRNA against ODIR1 or ODIR1 overexpression plasmid, following induced to differentiate into osteoblasts for 21 days. Differentiation was verified by ARS and ALP staining. $\mathbf{b}$ Representative images of bone formation (in Bio-Oss Collagen scaffolds) in ODIR1 and vector groups were detected by micro-CT. Scale bar, $0.1 \mathrm{~mm}$. c The BV/TV (bone volume/ tissue volume, \%) and BMD (bone mineral density, mg/cc) in ODIR1 and vector groups were analyzed by Xcapture Software. $\mathbf{d}$ Masson staining analyzes for collagen fiber. $\mathrm{H}$ and E staining analyzes for osteoid and IHC analyzes for OCN and OSX in bone matrix of ODIR1 and vector groups. Scale bar, $100 \mu \mathrm{m}$. 
volume/tissue volume) and BMD (bone mineral density) in the ODIR1 group showed 30 and 50\% decrease compared to that in the vector group, respectively (Fig. 2c). The Masson Trichrome staining results showed that less bone matrix (blue) was found in the ODIR1 overexpression group (Fig. 2d). $\mathrm{H}$ and $\mathrm{E}$ staining revealed that less newly formed bone and osteoid were observed in the ODIR1 group compared to vector group (Fig. 2d). IHC staining revealed that the intensity and range of OCN and OSX stained in osteoblasts of the ODIR1 group were less than those in the vector group (Fig. 2d), suggesting that ODIR1 inhibited the osteogenic differentiation of hUC-MSCs and the expression of OCN and OSX in vivo.

\section{ODIR1 inhibits the osteogenic differentiation of hUC-MSCs through OSX}

As Fig. 1e shown, the expression of the crucial osteogenic transcription factors RUNX2 and OSX were increased, while ODIR1 expression was decreased during the osteogenic differentiation of hUC-MSCs. To explore whether ODIR1 regulates the expression of OSX or RUNX2, we performed RT-qPCR and western blotting after ODIR1 ectopic expression or knockdown. After ODIR1 overexpression, OSX mRNA and protein levels were significantly reduced, while RUNX2 was not altered in the osteogenic differentiation of hUC-MSCs (Fig. 3a). Correspondingly, the OSX mRNA and protein were significantly increased in the ODIR1 knockdown group, while RUNX2 was also not altered in hUC-MSCs (Fig. $3 b)$.

To further determine how ODIR1 functions in the osteogenic differentiation of hUC-MSCs through OSX, RNA pulled down assay and mass spectrometry analyses were performed. The pull-down proteins were subjected to SDS-PAGE electrophoretic analysis, and the differential bands were analyzed by Mass Spectrum analysis (MS) (Fig. 3c). The MS results suggested that ODIR1 physically interacted with multiple proteins (Table S2). The GO functional annotation of these proteins is indicated in Table S3, and the data suggested that these proteins mainly involved in exocytosis, secretion and export from cells, single-stranded RNA and DNA binding. Epigenetic modification of histone $\mathrm{H} 2 \mathrm{~A}, \mathrm{H} 2 \mathrm{~B}, \mathrm{H} 3$, and $\mathrm{H} 4$ proteins is associated with gene transcription, and histone mark $\mathrm{H} 2 \mathrm{~B}$ mono-ubiquitination at lysine 120 (H2BK120ub) increased the level of $\mathrm{H} 3$ trimethylation at lysine 4 $(\mathrm{H} 3 \mathrm{~K} 4 \mathrm{me} 3)^{21,22}$. Further western blotting assay verified the ODIR1 pull-down proteins in hUC-MSCs lysates (Fig. 3d). RIP assays also confirmed the physical association between FBXO25, BARD1 or CUL3, and ODIR1 (Fig. 3e). Taken together, ODIR1 physically associates with the E3 ligases FBXO25, BARD1, CUL3 and the histone proteins $\mathrm{H} 2 \mathrm{~A}, \mathrm{H} 2 \mathrm{~B}$, and $\mathrm{H} 4$.

\section{ODIR1 inhibits OSX transcription by altering the enrichment of H2BK12Oub and H3K4me3 on the OSX promoter region}

ODIR1 knockdown increased the levels of H2AK119ub, H2BK120ub, and H3K4me3 (Fig. 3f), while ODIR1 overexpression decreased the expression of those proteins (Fig. 3g). It is known that lncRNAs regulate gene expression related to cellular activities through transcriptional or post transcriptional regulation ${ }^{23}$. H2BK120 ubiquitination can lead to transcriptional activation of target genes ${ }^{24}$, and it also promotes the tri-methylation of histone H3 at lysine 4 (H3K4me3). H3K4me3 modification was enriched on activated promoters and promotes the transcription of target gene ${ }^{25}$. Therefore, we first examined whether ODIR1 caused the fold enrichment of these histone epigenetic marks at the OSX or RUNX2 promoter region. Firstly, hUC-MSCs were incubated with OM for 7 days and the RNA levels of ODIR1 were decreased (Supplemental Fig. 5A), and the ChIP-qPCR results showed that low ODIR1 expression led to a significant increase enrichment of histone marks H2AK119ub (4.6 fold), H2BK120ub (3.7 fold), and H3K4me3 (4.3 fold) at the OSX but not at the RUNX2 promoter region (Fig. 4a, b). Moreover, knockdown of ODIR1 in hUC-MSCs (Supplemental Fig. 5B) also resulted in an increase in fold enrichment of H2AK119ub (4.2 fold), H2BK120ub (3.2 fold), and H3K4me3 (1.6 fold) at the promoter region of OSX but not RUNX2 in hUCMSCs (Fig. 4c, d). These data showed that ODIR1 inhibited osteogenesis by regulating OSX rather than RUNX2 in transcription level.

To further confirm the role of ODIR1 in the transcription of OSX, we detected the luciferase activities of the OSX promoter region using a dual luciferase reporter assay. The luciferase activity of the OSX promoter was markedly decreased while the activity of the RUNX2 promoter was not altered in the ODIR1 overexpression group (Fig. 4e). The luciferase activity of the OSX promoter also increased in the ODIR1 knockdown group (Fig. 4f). In addition, we found the luciferase activity of OSX promoter decreased in an ODIR1 dose-dependent pattern (Fig. 4g).

\section{ODIR1 inhibits H2B mono-ubiquitination through FBXO25 ubiquitination}

To explore the definite mechanism of H2BK120 monoubiquitination, we explored the relationship among the above three E3 ligases (FBXO25, CUL3, and BARD1) and H2AK119ub, H2BK120ub, and H3K4me3. Firstly, after hUC-MSCs were cultured in OM for 7, 14, and 21 days, the natural histones $\mathrm{H} 2 \mathrm{~A}, \mathrm{H} 2 \mathrm{~B}$ and $\mathrm{H} 3$ were not significantly altered, while the modified status H2AK119ub, H2BK120ub, and H3K4me3 were upregulated (Supplemental Fig. 6A). The proteins level of FBXO25 was 

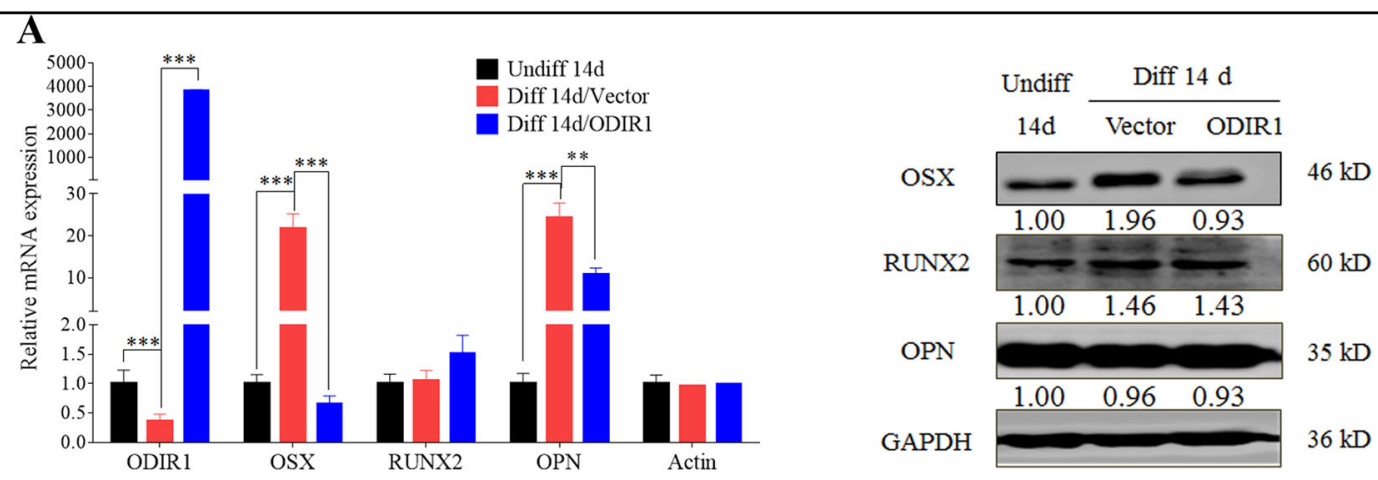

$\mathbf{B}$
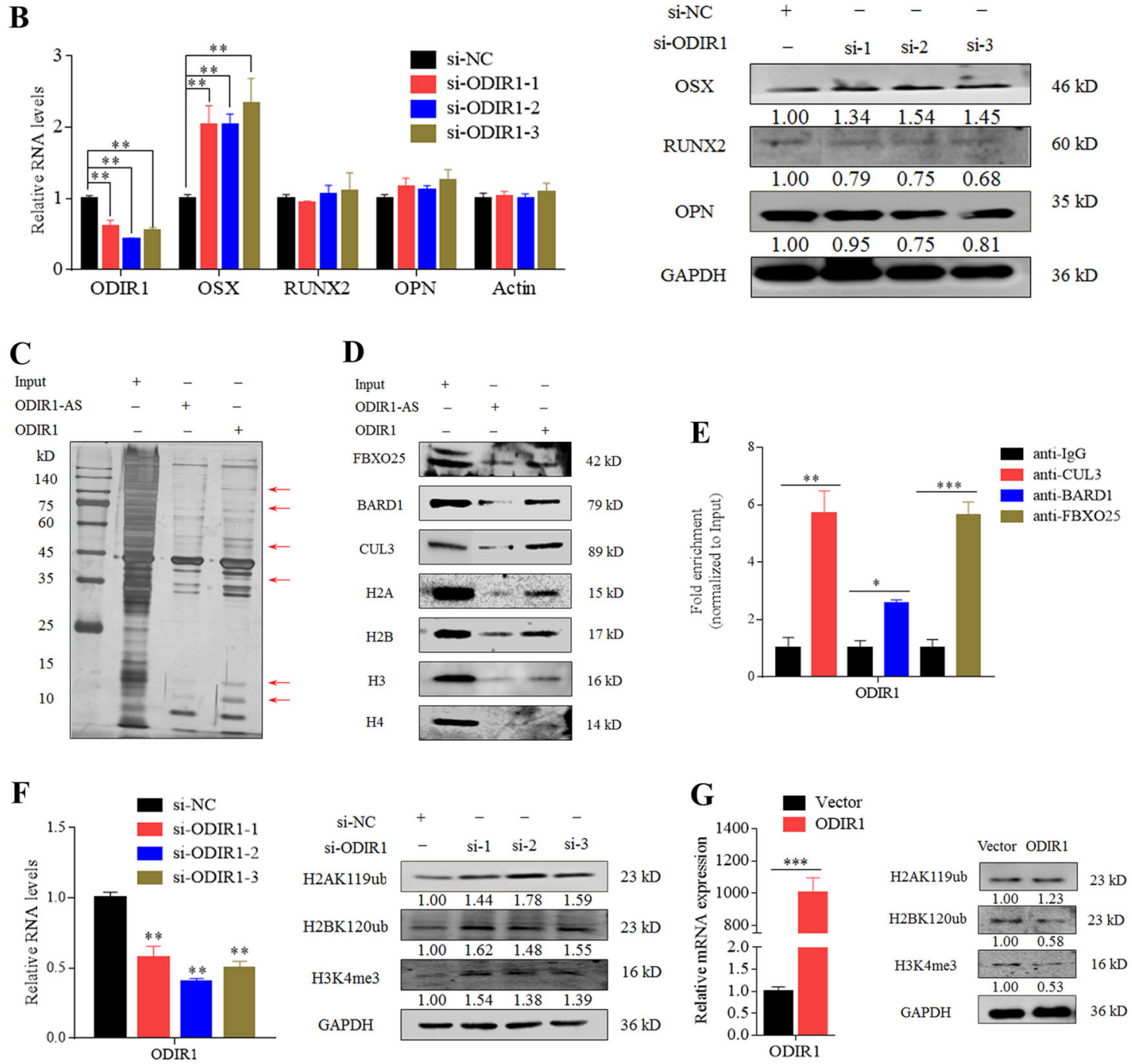

Fig. 3 ODIR1 physically interacts with E3 ligases and histone markers. a The hUC-MSCs were differentiated for 14 days and transfected with vector and ODIR1 plasmid. After $24 \mathrm{~h}$ transfection, RT-qPCR analysis for ODIR1, OSX, RUNX2, and OPN RNA levels, western blotting analysis for OSX, RUNX2, and OPN proteins levels. b The hUC-MSCs were transfected with NC and ODIR1 siRNAs, RT-qPCR analysis for ODIR1, OSX, RUNX2, and OPN RNA levels, western blotting analysis for OSX, RUNX2, and OPN proteins levels. c Biotin-labeled ODIR1 sense and anti-sense chains were incubated with hUC-MSCs lysates, and enriched products were collected and subjected to SDS-PAGE polyacrylamide gel electrophoresis and silver staining. Differential bands were identified by LC-MS analysis. d ODIR1 associated with FBXO25, BARD1 and CUL3, histone proteins including H2A, H2B, H3, and $\mathrm{H} 4$ as shown by RNA pull-down and western blotting. e ODIR1 was enriched by FBXO25, BARD1, and CUL3 in hUC-MSCs lysates. Anti-lgG were used as negative control. The fold enrichment values were normalized to that of Input. $\mathbf{f}$ The hUC-MSCs were transfected with siNC and ODIR1 siRNAs, and the RNA levels of ODIR1 was measured by RT-GPCR, the proteins levels of H2AK119ub, H2BK120ub and H3K4me3 were analyzed by western blotting assay. $\mathbf{g}$ The hUC-MSCs were transfected with vector and ODIR plasmid, and the RNA levels of ODIR1, OSX, and FBXO25 was measured by RT-qPCR, the proteins levels of H2AK119ub, H2BK120ub, and H3K4me3 were analyzed by western blotting assay. 

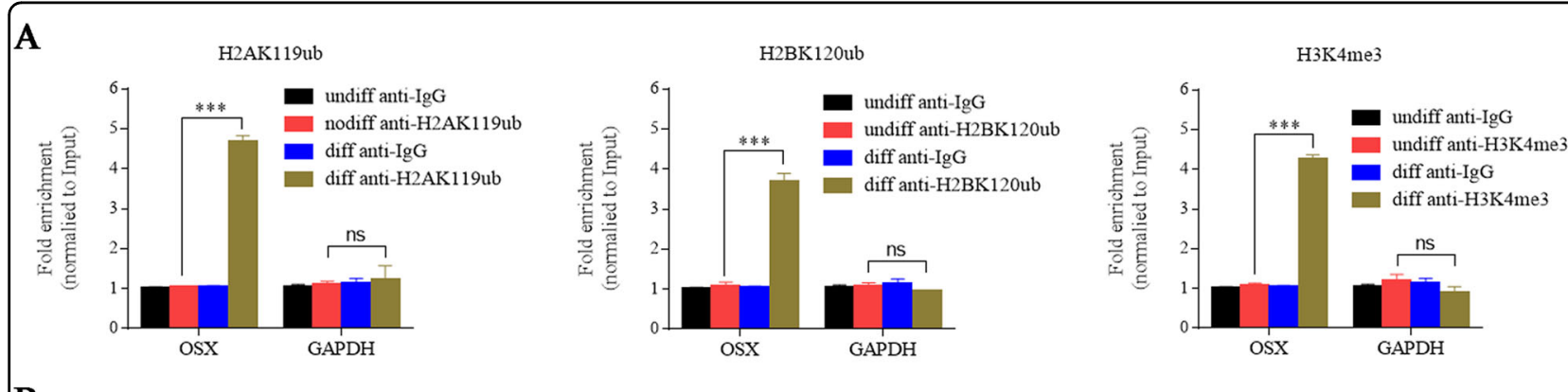

B
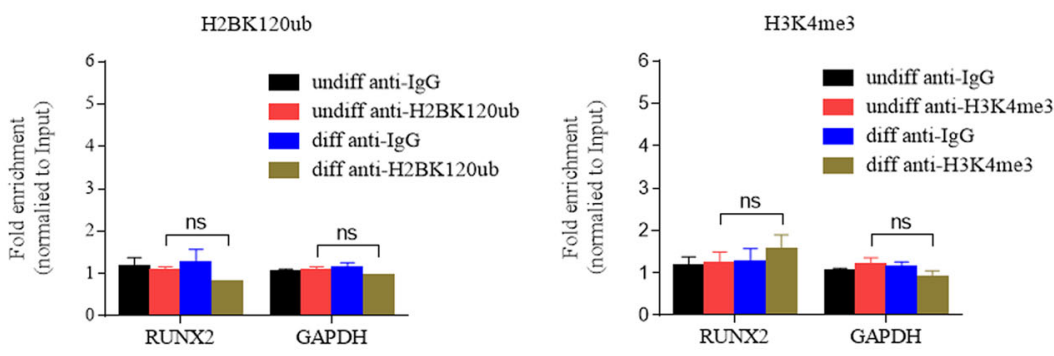

C

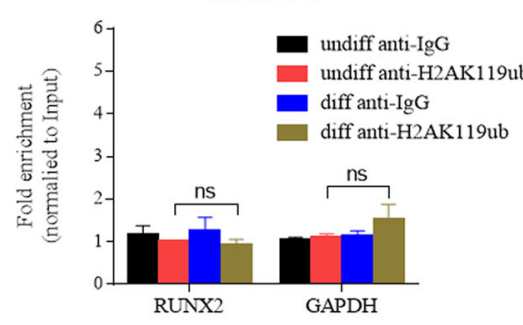

C H2AK119ub
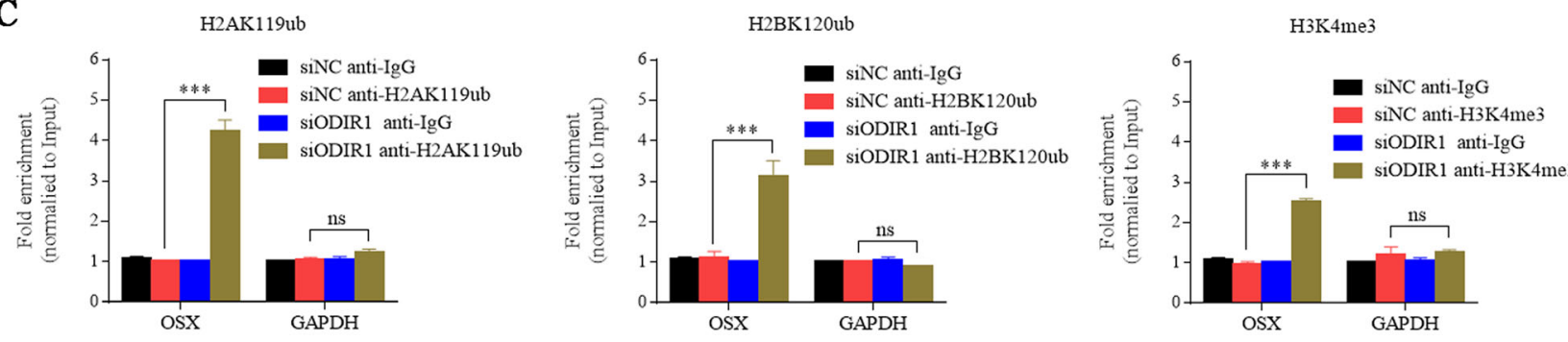

D

D H2AK119ub
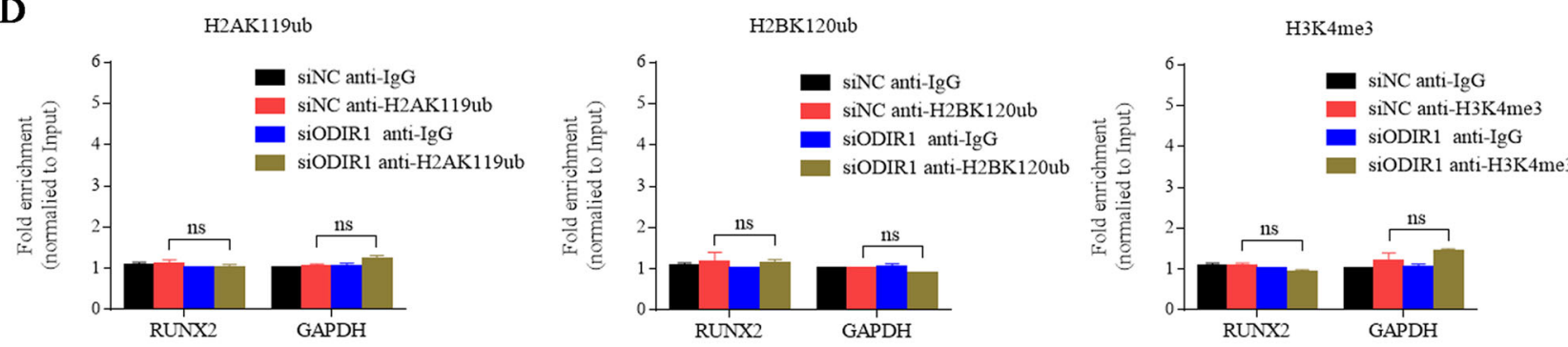

E

F



G - pcDNA-ODIR $1+\mathrm{pMIR}$

- pcDNA-ODIR1-0.1ug+pMIR-OSX-Luc - pcDNA-ODIR1-0.2ug+pMIR-OSX-Luc DeDNA-ODIR1-0.5ug+pMIR-OSX-Luc - pcDNA-ODIR1-1.Oug+pMIR-OSX-Luc
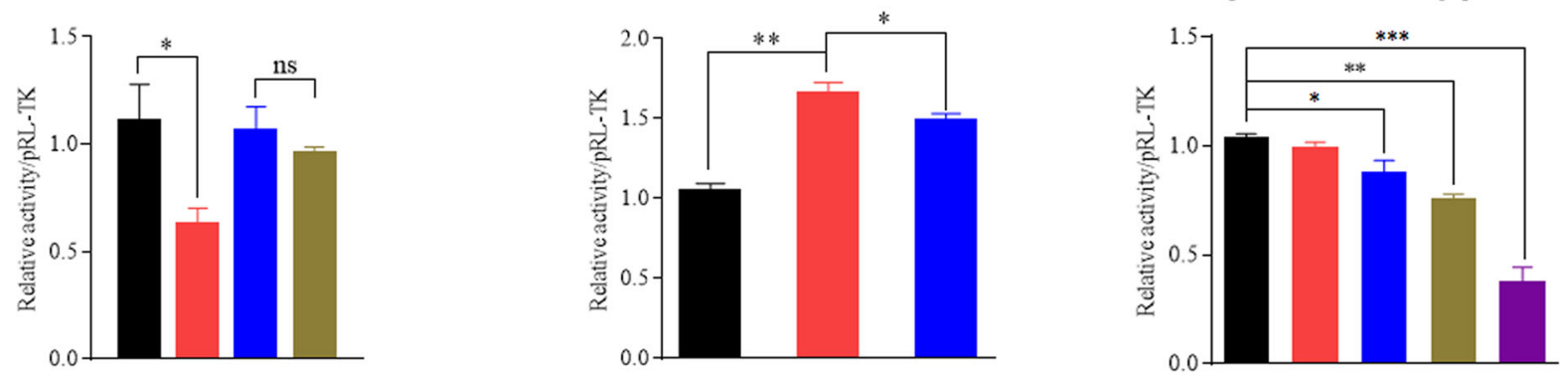

Fig. 4 (See legend on next page.) 
(see figure on previous page)

Fig. 4 ODIR1 alters the modification of histone marks on OSX promoter. $\mathbf{a}$, $\mathbf{b}$ The hUC-MSCs were cultured in normal growth medium (Undiff) or in osteogenic differentiation medium (Diff) for 10 days. The antibodies H2AK119ub, H2BK120ub, H3K4me3, and IgG were incubated with cell lysates, respectively, and the enrichment of those three histone markers on OSX or RUNX2 promoter was analyzed by ChIP-qPCR. c, d The hUC-MSCs were transfected with NC and ODIR1 siRNAs. The antibodies H2AK119ub, H2BK120ub, H3K4me3, and IgG were incubated with cell lysates, respectively, and the enrichment of those three histone markers on OSX or RUNX2 was analyzed by ChIP-qPCR. e The pMIR-OSX-luc (containing OSX promoter region) or pMIR-RUNX2-luc (containing RUNX2 promoter region) plasmids were co-transfected with pCDH-ODIR1 or pCDH plasmids into 293 cells, respectively, and the cell lysates were analyzed by luciferase assay. $\mathbf{f}$ The pMIR-OSX-luc plasmid was co-transfected with NC and ODIR1 siRNAs into 293 cells, respectively, and the cell lysates were analyzed by luciferase assay. $\mathbf{g}$ The pMIR-OSX-luc plasmids were co-transfected with different amounts $(0.1,0.2,0.5,1.0 \mu \mathrm{g})$ of pCDH-ODIR1 plasmids into 293 cells, respectively, and analyzed by luciferase assay.

gradually increased (Supplemental Fig. 6A). FISH analysis suggested that ODIR1 and FBXO25 mainly co-located at cell nucleus (Supplemental Fig. 6B). Moreover, ODIR1 decreased the protein levels of FBXO25 and CUL3 but did not affect BARD1 protein levels (Supplemental Fig. 6C, D). After transfection with specific siRNAs against BARD1, CUL3 and FBXO25, the expression of OSX was decreased while H2AK119ub was not changed; only knockdown of FBXO25 decreased the levels of H2BK120ub and H3K4me3 (Fig. 5a-c). FBXO25 (F-box protein 25 ), a member of the F-box protein family, is one of the four subunits of the SKP1-cullin-F-box (SCF) ubiquitin ligase complex ${ }^{26}$. CUL3 (Cullin 3), an E3 ubiquitin ligase, mediates the ubiquitination of target proteins such as H2A, through the Cullin-RING-based BCR complex ${ }^{27}$. Another E3 ligase of the Cullin family, Cul4, which forms a Clr4-Rik1-Cul4 complex and recruits $\mathrm{H} 2 \mathrm{~B}$ and $\mathrm{H} 4$ to form RNAi-mediated heterochromatin ${ }^{28}$. BARD1 (BRCA1 associated RING domain 1) and BRCA1 forms an E3 ubiquitin-protein ligase heterodimer that mediated $\mathrm{H} 2 \mathrm{~A} / \mathrm{H} 2 \mathrm{~B}$ ubiquitination and regulated the differentiation of mesenchymal stem cells ${ }^{29}$. In conclusion, CUL3, BARD1, and FBXO25 are associated with the ubiquitination of histone proteins. In addition, we found that knockdown of BARD1 and CUL3 did not affect the RNA levels of FBXO25 while knockdown of CUL3 increased the protein level of FBXO25 (Fig. 5d, e). Given that CUL3 is an E3 ligase, we next determined whether CUL3 could reduce $\mathrm{FBXO} 25$ protein levels by promoting its ubiquitination and degradation. As expected, CUL3 promoted FBXO25 degradation through the proteasome pathway (Fig. 5f, g).

In addition, as the amount of ODIR1 transfection increased, the inhibition of FBXO25 and H2BK20ubi increased gradually; as the amount of transfection of FBXO25 increases, the protein levels of FBXO25 and H2BK20ubi also increase gradually. Moreover, ectopic expression of ODIR1 reduced the ubiquitination of H2BK120 protein and shortened the H2BK120ub protein half-life (Fig. 6c, d). Ectopic expression of FBXO25 did not affect the expression or half-life of exogenous $\mathrm{H} 2 \mathrm{~B}$ protein but increased the protein levels of H2BK20ubi (Fig. 6e, f). We then performed a denaturing immunoprecipitation assay to investigate whether $\mathrm{FBXO} 25$ affects the ubiquitination levels of H2BK120ub. The data revealed that FBXO25 increased the levels of H2BK120ub (Fig. 6g), however, the increase of H2BK120ub was obviously reversed by ODIR1 overexpression (Fig. 6h). These results indicate that ODIR1 inhibits FBXO25-mediated H2B ubiquitination.

\section{Discussion}

MSCs differentiation into osteoblasts is a multiple-step and extremely complicated process, and the BMP-Smad signaling pathway is one of the regulatory axis in this process $^{30}$. When bone morphogenetic protein (BMPs) is activated and targets Smad family members, SMADs are phosphorylated and sequentially facilitated the transcription of the osteogenesis factors RUNX2 and OSX and then activated the expression of downstream osteoblast markers, such as OCN, OPN, and Collal ${ }^{31,32}$. In this process, RUNX2 mediated MSCs differentiation into osteochondro-progenitors and simultaneously activated OSX and ATF4 during the osteochondro-progenitor differentiation into immature osteoblasts, mature osteoblasts and finally matured into osteocytes ${ }^{31-33}$. RUNX2 (CBFA; core-binding factor-alpha) is a member of the runthomology domain protein family ${ }^{34}$. OSX is an essential transcription factor contains a zinc-finger domain ${ }^{32}$. Phosphorylation of RUNX2 activated the transcription of OSX during osteogenesis of stem cells ${ }^{35}$. In this study, we found that IncRNA ODIR1 downregulated while both RUNX2 and OSX upregulated during the osteogenic differentiation of hUC-MSCs (Fig. 1e).

As shown, ODIR1 could recruit FBXO25, BARD1, and CUL3, while only FBXO25 and CUL3 could regulate the levels of H2BK120ub and H3K4me3, which suggested that FBXO25 and CUL3 might be involved in histone modification (Fig. 3c, d). FBXO25 is a member of the F-boxing protein (FBP) family contains an ubiquitination target binding domain, and is one subunit of SCFs (Skp1-Cul1FBP) complex ${ }^{26}$. FBP is responsible for recruiting substrates to SCF complex, and then binding to the E3 ligase members SCF, Skp1, and Cul1; finally, ubiquitinating 


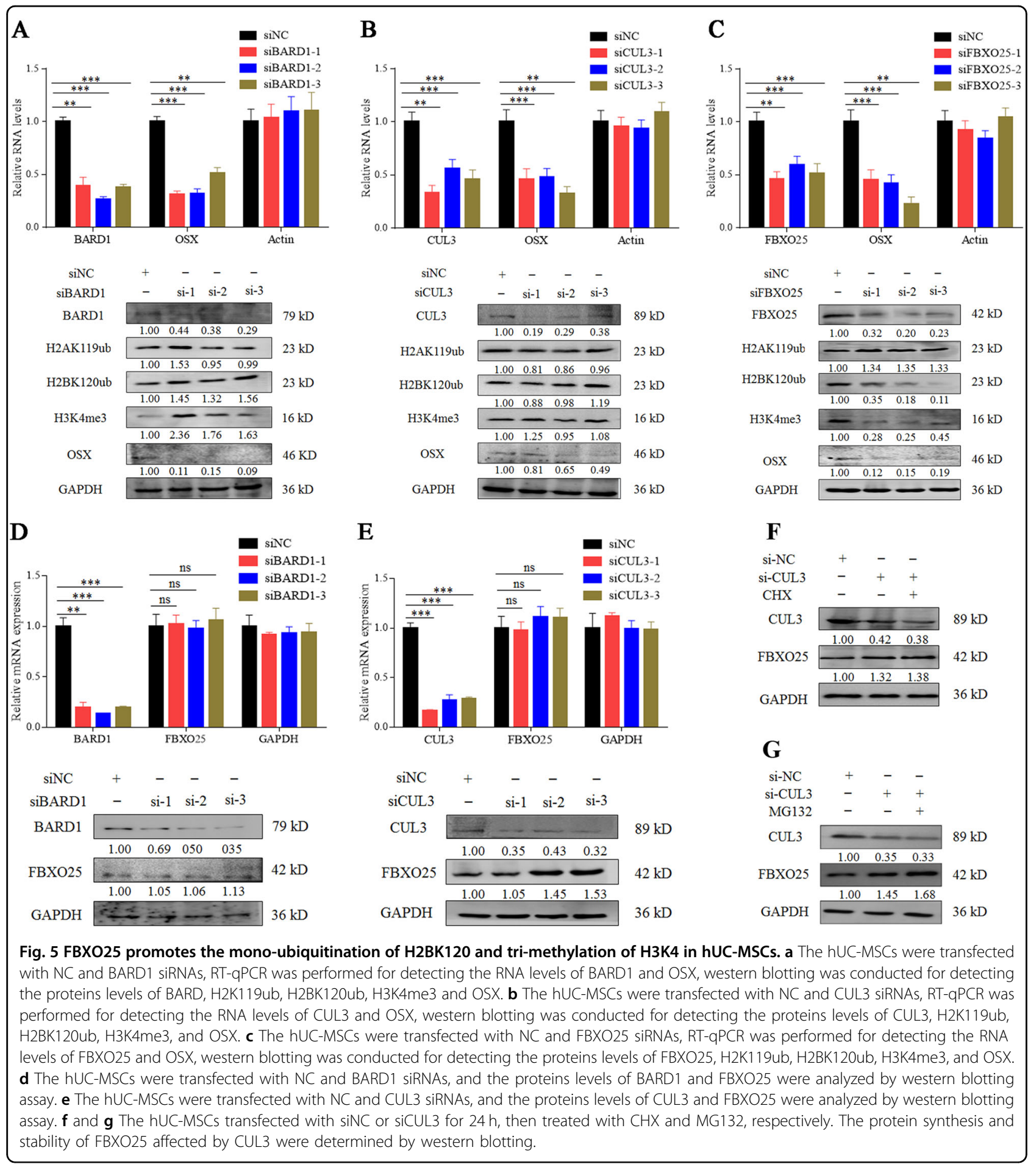

specific substrates under physiological or pathological process $^{36}$. It has been found that FBXO25 acts as a novel E3 ligase to ubiquitinate the cardiac transcription factors Isl1, Nkx2-5, and Hand1, and is involved in hypertrophic cardiomyocyte growth and cardiogenesis ${ }^{37}$. In addition, FBXO25 decreased the active levels of phosphorylated ERK1/2 through its E3 ligase SCF1 activity ${ }^{38}$. As an E3 ubiquitin ligase, FBXO25 promotes proteins degradation mainly through the ubiquitination pathway, while the mechanism by which FBXO25 regulates histone marks through epigenetic modification was elusive. In this study, we found that $\mathrm{FBXO} 25$ increased the monoubiquitination of H2BK120 and subsequently the trimethylation of H3K4 during osteogenesis (Fig. 5c), which 


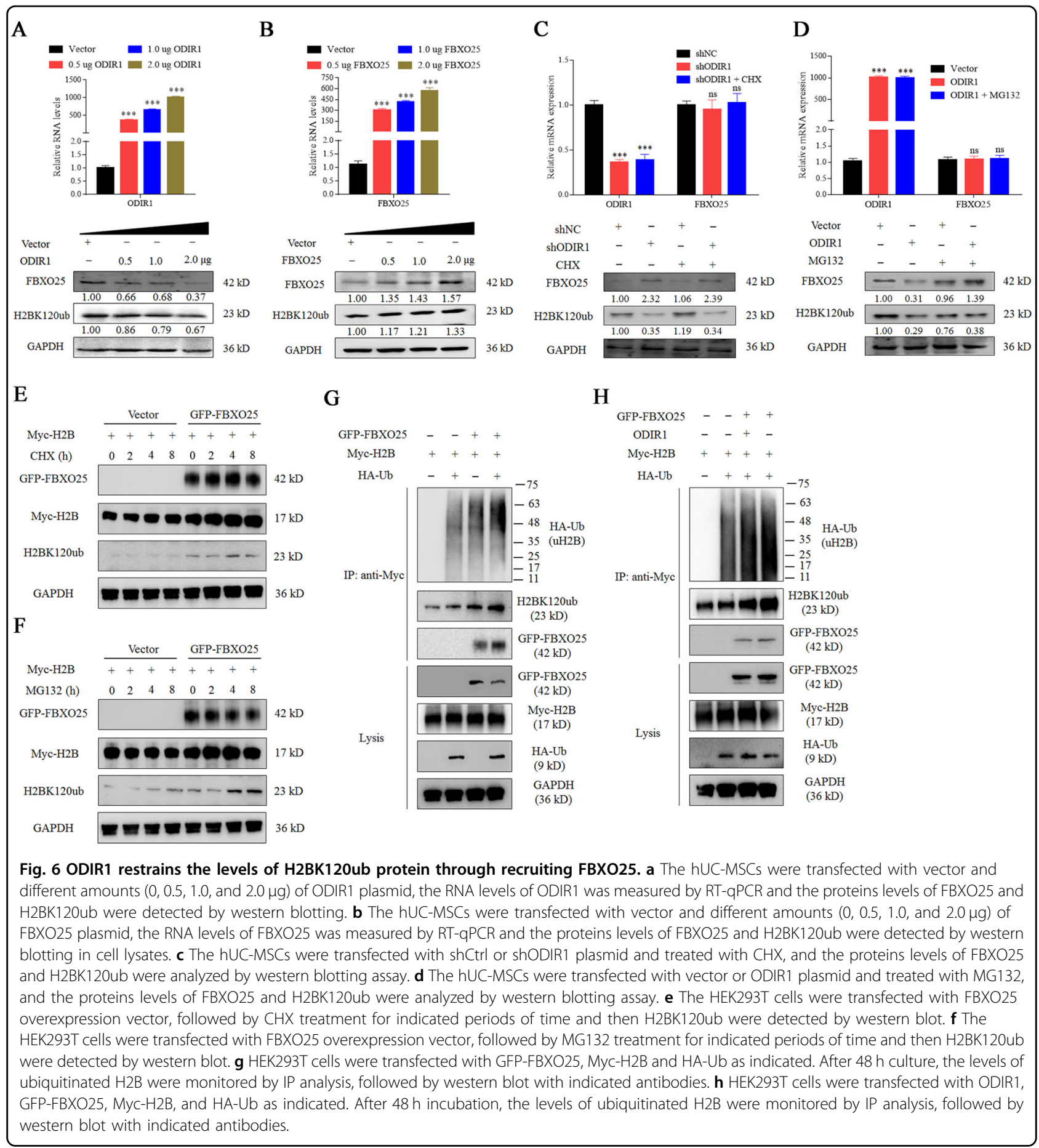

indicated that FBXO25 regulates the histone modification during hUC-MSCs differentiation.

During the osteogenic differentiation of WJ-MSCs, the transcription of RUNX2 is strongly activated, and that of OSX is inactivated. The mechanism suggested that high activity of the activating histone marks H3K27Ac, H3Ac, H3K4me1 and H3K4me3 occupancies on RUNX2 promoter region, while the high activity of inhibitory histone marks H3K27me3 and H3K9me3 was enriched at the promoter region of $\mathrm{OSX}^{39}$. In our study, we found that the activities of H2AK119ub (histone H2A monoubiquitinated at lysine 119), H2BK120ub (histone H2A monoubiquitinated at lysine 120) and H3K4me3 (histone H3 trimethylated at lysine 4) were significantly increased during the osteogenic differentiation of hUC-MSCs (Fig. S6A). In mammalian cells, H2A mono-ubiquitination is 


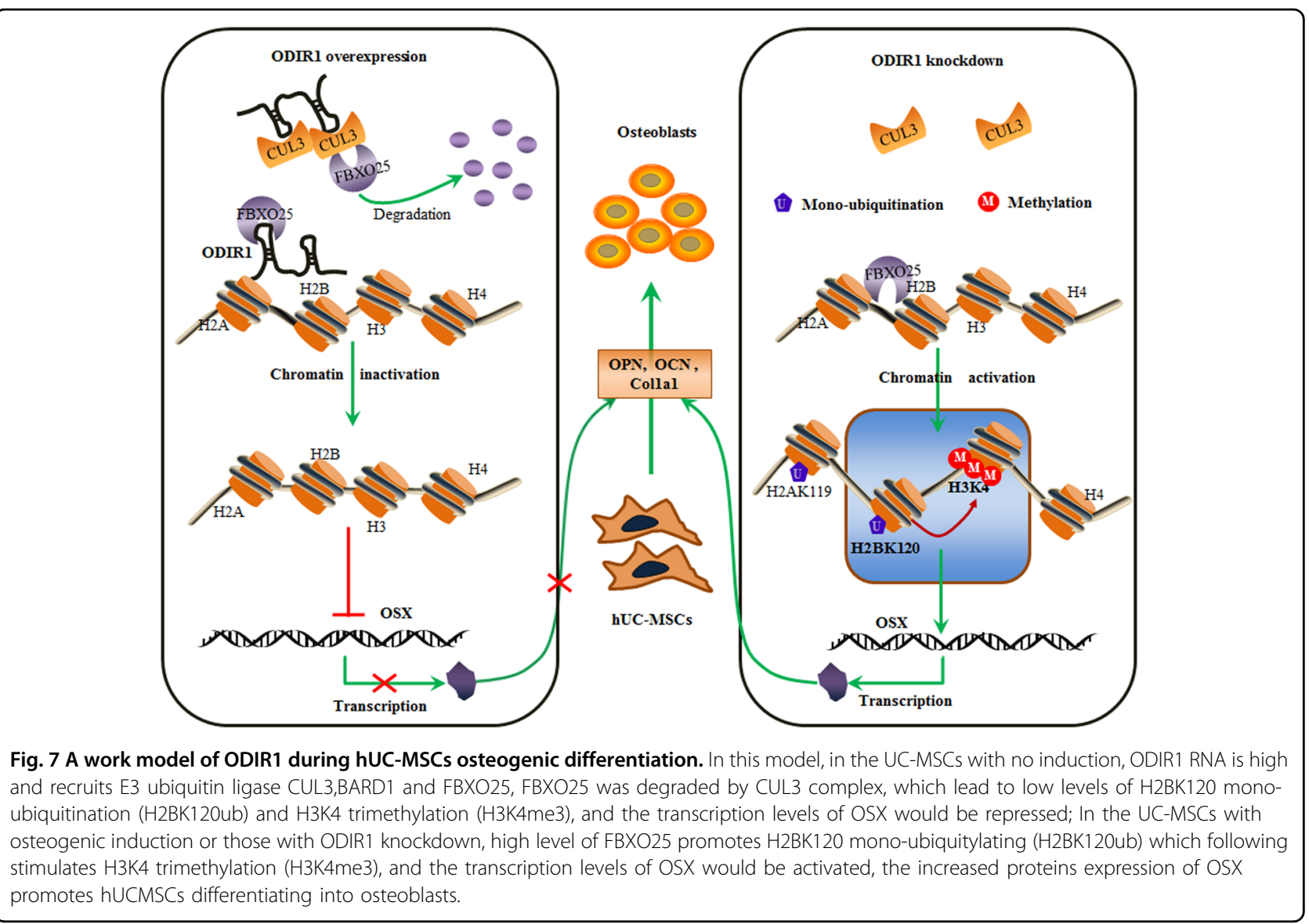

mediated by polycomb repressive complex 2 (PRC2) and is related to transcriptional repression ${ }^{40,41}$. CRL4B (Cullin4B-Ring E3 ligase complex) physically interacts with PRC2 and then attenuates the activity of H2AK119ub and H3K27me3 (transcriptional inactivated marks) ${ }^{42}$. Contrary to H2AK119, H2BK120ub is an activating histone mark, and ubiquitination is mediated by the ubiquitinconjugating enzyme RAD6 ${ }^{40,43}$. H2BK120ub attenuates MEIS1-mediated apoptosis ${ }^{44}$ or maintains the pluripotency of stem cells by promoting the expression of pluripotential genes ${ }^{45}$. During mouse embryonic stem cells (ESCs) differentiation, H2BK120ub was preferentially abounded on the coding region of differentiation-related transcription factors OCT4, Sox17, Nanog, etc, and the decrease in H2BK120ub affects their transcription, triggering further transforms in ESC differentiation ${ }^{46}$. High expression of USP44 maintains the low levels of H2Bubi in undifferentiated mouse ESCs; when cells in an efficient differentiated state, USP44 levels decreased while H2BK120ub increased. Mechanistically, depletion of USP44 prior to the differentiation signal promotes H2Bubi expression; however, impairing the dynamic turnover of H2BK120ubinterferes with proper execution of the differentiation process ${ }^{47}$. In eukaryotes, H3K4me3 is an activating histone mark that is associated with gene expression and active chromatin, and can be methylated (mono-methylated, di-methylated, or tri-methylated) by SET7 or demethylated by JARID1A ${ }^{48,49}$. Several studies have reported that $\mathrm{H} 2 \mathrm{~B}$ mono-ubiquitination can facilitate H3K4 trimethylation on chromatin. H2BK120ub mediated by Set1-COMPASS can promote the activities of H3K4me $3^{50}$. hRAD6ubiquitylates H2BK120 on chromatinized via direct interaction with hPAF-bound hBRE1and the latter directly stimulated H3K4 dimethylation or tri-methylation ${ }^{21}$. During myogenic differentiation, the RNF20 mediated histone talk between H2BK120ub and H3K4me3 is decreased, which demonstrated that Set1-dependent H3K4me3 was inhibited by the decreased interaction with $\mathrm{H} 2 \mathrm{BK} 120 \mathrm{ub}^{22}$. In this study, we found that ODIR1 reduced the occupancies of H2BK120ub and H3K4me3 on the OSX promoter (Fig. 4). In ODIR1 knockdown hUC-MSCs, we found increased occupancy of inhibitory mark H2AK119ub at the OSX promoter, while the transcription of OSX was increased instead of inhibited, which suggested that the activation effect of H2BK120ub coordinate with H3K4me3 on the OSX promoter region may be slightly stronger than the inhibition effect of H2AK119ub. According to the 
experimental results, during osteogenic differentiation, OSX is the early osteogenic transcription factor during osteochondro progenitor differentiation into immature osteoblasts and the subsequent osteoblasts maturation process. In summary, the dynamic changes in OSX level might contribute to the multi-step osteogenic differentiation process of MSCs.

LncRNAs are involved in epigenetic regulation by recruiting related essential molecules to the methylated or ubiquitinated protein, and then exerts their biological functions via transcriptional activation or repression ${ }^{51}$. Overall, we propose a tentative work model of ODIR1 during osteogenic differentiation of hUC-MSCs (Fig. 7). When ODIR1 expression is high level in hUC-MSCs, it recruits the E3 ubiquitin ligase CUL3, which induces the degradation of FBXO25 and then suppresses the levels of H2BK120ub and subsequently H3K4me3 occupying on the OSX promoter region. After ODIR1 knockdown in hUC-MSCs, the decoy and inhibitory effects of ODIR1 on FBXO25 and H2BK120 are released, thus inducing H2BK120 mono-ubiquitination and subsequent H3K4 trimethylation enrichment on the OSX promoter region The transcription of OSX increases and promotes the transcription of downstream osteogenic genes, such as OPN, OCN, and Colla1, ultimately promoting hUC-MSCs differentiation into osteoblasts.

\section{Materials and methods}

\section{Cell culture and osteogenic induction}

The human umbilical cord-derived mesenchymal stem cell (hUC-MSCs) line QC1205 was obtained from the National Engineering Research Center of Human Stem Cells (Changsha, China) and maintained at $37^{\circ} \mathrm{C}$ in DME/ F12 (1:1) medium with 10\% FBS (Gibco, Grand Island, NT, USA). The QC1205 cell line was characterized using flow cytometry (BD, New Jersey, USA) for MSCs specific surface markers like CD44-FITC (99.7\%), CD73-PE (99.8\%), CD90-PerCP (99.7\%), CD29-PE (99.9\%), and CD34-APC (0.102\%) (Fig. S1). HEK293 and $293 \mathrm{~T}$ cell lines were cultured with DMEM medium (Gibco, Grand Island, NT, USA) with $10 \%$ FBS. The proliferation medium (PM) was DME/F12 medium with $10 \%$ FBS. The osteogenic differentiation medium (OM) was DME/F12 medium with $10 \%$ FBS supplemented with the following differentiation compositions: $50 \mathrm{mg} / \mathrm{L}$ ascorbic acid, $10 \mathrm{mM} \beta$-glycerophosphate, and $10^{-7} \mathrm{M}$ dexamethasone (Sigma, St. Louis, MO, USA). hUC-MSCs were induced for osteogenic differentiation in $\mathrm{OM}$ for $7,14,21$, or 28 days for further experiments.

\section{Energy dispersive spectroscopy (EDS)}

The samples for scanning electron microscopy (SEM) and energy dispersive spectroscopy (EDS) analyses were mounted on aluminum sample holders using double- sided and electrically conductive adhesive tape, followed by sputtering with gold for $30 \mathrm{~s}$ using a sputter coater (LJ16, Beijing Yulong Times Technology Co., China) to increase their electrical conductivity ${ }^{52,53}$. Then, the microstructure and elemental composition of the samples were characterized by a Scanning Election Microscope (PhenomProX, Phenom-World BV, Netherlands) equipped with an EDS (INCA, Oxford Instruments, UK) under an acceleration voltage of $15 \mathrm{kV}$.

\section{Microarray and bioinformatic analysis}

After 21 days of culture in PM or OM, hUC-MSCs were harvested and the IncRNA expression profile was obtained from OE Biotech (Shanghai, China) using Affymetrix WT (Thermo Fisher Scientific, Waltham, MA, USA). For the filtering of differentially expressed lncRNAs, fold change values $<-2.0$ and $>2.0$ and $p$-values at $<0.05$ were used for the analysis.

\section{Plasmid construction}

Full-length ODIR1 cDNA was amplified from hUCMSCs and cloned into $\mathrm{pCDH}$ vector (pCDH-ODIR1) for stable or transient expression of ODIR1, and the Fulllength ODIR1 was also cloned into the pcDNA3.1/His C vector (pcDNA3.1-ODIR1) for luciferase and RNA pull down assays. The shODIR1 sequences were synthetized by BBI Life Science (Shanghai, China) and subcloned into the pLVTH vector (pLVTH-shODIR1) for stable knockdown of ODIR1. The OSX and RUNX2 promoter regions were amplified from hUC-MSCs cDNA and subcloned into the pMIR vector (pMIR-OSX-Luc and pMIRRUNX2-Luc) for the luciferase assay. All plasmids were maintained in DH5 $\alpha$ competent cells, and plasmid DNA was extracted using Plasmid Mini Kit I (OMEGA, Norcross, USA) following the manufacturer's instructions.

\section{Cell transfection}

Target siRNAs (siODIR1, si-OSX, si-RUNX2, siBARD1, siCUL3, and siFBXO25) or negative control siRNA were obtained from GenePharma (Shanghai, China). SiRNA transfections were performed using $100 \mathrm{nM}$ siRNA and Lipofectamine 2000 Reagent (Invitrogen, Waltham, MA, USA) following the manufacturer's instructions. Plasmid transfections were performed using $2 \mu \mathrm{g}$ plasmid and Lipofectamine 2000 Reagent in 6-well plates according to the manufacturer's instructions. Total RNA and protein were extracted from hUC-MSCs after transfection for 24 and $48 \mathrm{~h}$, respectively.

\section{Stable cell line establishment}

The pCDH-ODIR1 $(10 \mu \mathrm{g})$ or pLVTH-shODIR1 $(\mu \mathrm{g})$ and corresponding control vector were co-transfected with two packaging vectors $(7.5 \mu \mathrm{g}$ pSPAX2 and $7.5 \mu \mathrm{g}$ pMD2G) into 293FT cells for $48 \mathrm{~h}$ to produce lentivirus. 
The supernatant medium containing target lentivirus was collected and filtrated by $0.22 \mu \mathrm{M}$ aperture PES membranes (Millipore, Darmstadt, Germany). Then hUCMSCs were infected with lentivirus mixed with 1:1000 Polybrene (Santa Cruz Biotechnology, CA, USA). The infection efficiency was detected by BX60 Fluorescence Microscope (Olympus, Japan) after $48 \mathrm{~h}$ of infection. The GFP positive cells were analyzed and sorted by Flow Cytometry and subsequently cultured for further experiments.

\section{Real-time quantitative PCR (RT-qPCR)}

Total RNA was extracted from QC1205 and 293FT cells using TRIzol reagent (Life Technologies, Carlsbad, CA, USA). Then reverse transcription (RT) of total RNA was conducted by RevertAid First Strand cDNA Synthesis Kit (Thermo Fisher Scientific, Waltham, MA, USA) using random primers. RT-qPCR was performed using $2 \times$ SYBR Green qPCR Master Mix (Biotool, HOU, USA) and a program conducted on Real-Time PCR CFX96 Instrument (Bio-Rad, Hercules, CA, USA). GAPDH and $\beta$-Actin were used as internal references for analyzing relative RNA levels. Relative RNA levels were obtained from the Bio-Rad CFX manager (Bio-Rad, Hercules, CA, USA). The primers are listed in Table S4, and all samples were performed in triplicate independent tests.

\section{Western blotting}

Cells were harvested and then lysed with RIPA lysis buffer containing $1.0 \mathrm{mM}$ protease inhibitor cocktail, $1.0 \mathrm{nM}$ DTT and PMSF for $30 \mathrm{~min}$ at $4{ }^{\circ} \mathrm{C}$. Then, the protein supernatant was collected using a microcentrifuge at $13,000 \mathrm{rpm}$ for $15 \mathrm{~min}$ at $4{ }^{\circ} \mathrm{C}$. Appropriate protein $(50 \mu \mathrm{g})$ of samples were subjected to $10-12 \%$ SDS-PAGE gel electrophoresis and electroblotted into PVDF membranes (Millipore, Darmstadt, Germany) as previously described $^{54}$. The membranes were blocked with skimmed milk solution (5\%) and incubated with the following primary antibodies: GAPDH (1:3000; Origene, USA), $\beta$-Actin (1:3000; Abclonal, USA), Osteocalcin (1:1000; Santa Cruz Biotechnology, USA), RUNX2 (1:1000; Proteintech, China), SP7/Osterix (1:1000; Abcam, USA), BARD1 (1:500; Santa Cruz Biotechnology, USA), FBXO25 (1:300; Santa Cruz Biotechnology, USA), CUL3 (1:500; Santa Cruz Biotechnology, USA), H2A (1:1000; Absin, China), H2B (1:1000; Abcam, USA), H3 (1:1000; CST, USA), H2AK119ub (1:1000; CST, USA), H2BK120ub (1:1000; CST, USA), and H3K4me3 (1:2000; CST, USA). The results were visualized using a MiniChemi 610 System Instrument (Beijing, China).

\section{In vitro ubiquitination assay}

To determine whether ODIR1 regulates the protein expression of FBXO25 at the post-transcriptional level, the proteasome inhibitor MG132 and the protein synthesis inhibitor cycloheximide (CHX; Sigma Aldrich, Shanghai, China) were applied to evaluate the protein stability or synthesis ability of $\mathrm{FBXO} 25$, respectively. hUC-MSCs transfected with negative vector or ODIR1 full length expression vector were cultured in PM containing MG132 (100 ng/ml) or cycloheximide (CHX) $(100 \mu \mathrm{g} / \mathrm{ml})$ for $8 \mathrm{~h}$, respectively. The hUC-MSCs with sh$\mathrm{NC}$ and sh-ODIR1 were incubated with PM containing MG132 (100 ng/ml) or cycloheximide (CHX) $(100 \mu \mathrm{g} / \mathrm{ml})$ for $8 \mathrm{~h}$, respectively. After treatment, the total protein was extracted for western blotting analysis. For the in vitro ubiquitination assays, HEK293T cells were transfected with GFP-FBXO25, Myc-H2B and HA-Ub or ODIR1 as indicated. After $48 \mathrm{~h}$ of culture, the levels of ubiquitinated H2B were monitored by IP analysis, followed by western blotting with the indicated antibodies.

\section{Immunofluorescence and in situ hybridization}

A total of $5.0 \times 10^{3}$ hUC-MSCs cells were seeded in 6well plates for $24 \mathrm{~h}$. Then, the cells were fixed with $4 \%$ PFA (paraformaldehyde; Bioshrap, China) solution and the following steps were monitored as previously described $^{55,56}$. The images were visualized and collected using UltraVIEW VoX (PerkinElmer, Waltham, USA) confocal fluorescence microscope. In situ hybridization was carried out following the manufacturer's protocol. The synthetic ODIR1 Digoxin tag probe sequences (Table S4) were obtained from Sangon Biotech (Shanghai, China).

\section{Alizarin red $S$ (ARS) staining and ALP staining}

The hUC-MSCs were seeded in 6-well plates. Osteogenic induction was performed in $\mathrm{OM}$ when the cells reached $60-80 \%$ confluence, and the negative control cells were cultured in PM. After 21 days of osteogenic differentiation, cells were fixed with 4\% PFA solution and ARS staining was performed using the ARS Kit (Sigma, USA) following the manufacturer's instructions. After osteogenic differentiation for 14 days, cells were fixed with $4 \%$ PFA solution and ALP staining was performed using the ALP Kit (Beyotime, Shanghai, China) and following the manufacturer's instructions.

\section{RNA pull-down assay}

Full length ODIR1 RNA (ODIR1) and ODIR1-antisense RNA (ODIR1-AS) were transcribed in vitro from pcDNA3.1-ODIR1 and pcDNA3.1-ODIR1-AS and labeled with biotin by the Biotin RNA Labeling Mix (Sigma, USA) and T7 RNA polymerase (Sigma, USA). The biotinlabeled RNAs $(3 \mu \mathrm{g})$ was subjected to heat shock at $90^{\circ} \mathrm{C}$ for $2 \mathrm{~min}$ and cooled at room temperature (about $25.0^{\circ} \mathrm{C}$ ) for $20 \mathrm{~min}$, folded with RNA structure buffer, then mixed with cell extract of hUC-MSCs, incubated with Streptavidin Agarose Resin (Thermo Fisher Scientific Inc., 
Waltham, MA, USA) at $4{ }^{\circ} \mathrm{C}$ for $2 \mathrm{~h}$ and washed. The retrieved proteins were subjected to SDS-PAGE gel electrophoresis and the protein band of the ODIR1 group was subjected to silver staining, and differential protein bands were identified using high resolution mass spectrometry (LC-LTQ-Orbitrap; Thermo Fisher Scientific, Waltham, MA, USA). The identified proteins were examined using regular western blotting assay. The GO functional annotation was analyzed using the Gene Ontology Consortium (http://www.geneontology.org/).

\section{RNA immunoprecipitation (RIP)}

The cells extract of hUC-MSCs was admixed with Protein-G/A Plus Agarose (Santa Cruz Biotechnology, USA), and then incubated with $2 \mu \mathrm{g}$ antibodies against FBXO25, CUL3, BARD1 or normal rabbit anti-IgG at $4{ }^{\circ} \mathrm{C}$ for $4 \mathrm{~h}$ and washed. Then, the samples were subjected to RT-qPCR. The fold enrichment of precipitated ODIR1 RNA was examined by RT-qPCR. The primers used are listed in Table S4. All samples were detected in triplicate.

\section{ChIP}

The hUC-MSCs were induced with OM for 7 days or were transfected with siODIR1 for $48 \mathrm{~h}$. Then the cells were cross-linked with $1 \%$ PFA solution for $10 \mathrm{~min}$ at $37^{\circ} \mathrm{C}$ and the cross-linked media were neutralized with $0.125 \mathrm{M}$ Glycine (Bioshrap, China) for $10 \mathrm{~min}$ at room temperature $\left(\sim 25^{\circ} \mathrm{C}\right)$. Then, cells were harvested and lysed by SDS lysis buffer at $4{ }^{\circ} \mathrm{C}$ with rotation vortexing for $30 \mathrm{~min}$, and the supernatant lysate was sonicated using Cole-Parmer Instruments CP130 (Vernon, Illinois, USA) for $15 \mathrm{~min}$ at the frequency of $1.0 \mathrm{~s}$ ultrasound and $1.0 \mathrm{~s}$ stop (for one cycle). After centrifugation at $13,000 \mathrm{rpm}$ for $10 \mathrm{~min}$ at $4.0^{\circ} \mathrm{C}$, the supernatant was collected and mixed with the antibodies against H2AK119ub, H2BK120ub, and H3K4me3 (2 $\mu \mathrm{g}$ each antibody), incubated with ProteinA/G Immunoprecipitation Magnetic Beads (Santa Cruz Biotechnology, USA) for $2 \mathrm{~h}$ at $4.0^{\circ} \mathrm{C}$ and washed using wash buffer. The immunoprecipitated DNAs were decross-linked using 10\% Chelex-100 mixture (Bio-Rad, Hercules, CA, USA) for $10 \mathrm{~min}$ at $99.0^{\circ} \mathrm{C}$, and the DNA supernatant was collected by centrifugation at 13,000 rpm for $3 \mathrm{~min}$ at $4{ }^{\circ} \mathrm{C}$. The immunoprecipitated DNAs were detected by RT-qPCR using the OSX and RUNX2 promoter primers (Table S4). The relative fold enrichment of H2AK119ub, H2BK120ub and H3K4me3 at the OSX or RUNX2 promoter region was to the Input and the immunoprecipitation values were normalized to normal rabbit IgG.

\section{Luciferase assay}

HEK293T cells were collected and seeded at $1.0 \times 10^{5}$ cells in 24-well plates per well, and then pcDNA3.1-
ODIR1 was co-transfected with PMIR-OSX-Luc or pMIRRUNX2-Luc when the cells grow to $80 \%$ confluence. For further verification, the pcDNA3.1-ODIR1 and pcDNA3.1-NC, siODIR1 and siNC plasmids were cotransfected with pMIR-OSX-Luc into 293T cells. For ODIR1 degree dependent luciferase assay, different amounts $(0.1,0.2,0.5$, and $1.0 \mu \mathrm{g})$ of pcDNA3.1-ODIR1 were co-transfected with pMIR-OSX-Luc into $293 \mathrm{~T}$ cells. The cells of each well were co-transfected with pRL-TK (Renilla), which was used as internal reference. The dualluciferase assays were monitored using the DualLuciferase Reporter Assay System (Promega, WI, USA) following the manufacturer's instructions. All samples were detected in triplicate independent tests.

\section{Bone formation in vivo}

The stable overexpression of ODIR1 in hUC-MSCs was induced in $\mathrm{OM}$ at $37^{\circ} \mathrm{C}$ for 7 days. Then the cells were harvested and placed on the surface of Bio-Oss Collagen scaffolds $\left(5 \times 5 \times 1.75 \mathrm{~mm}^{3}\right)$ (Geistlich; Pharma, Australia) at $37^{\circ} \mathrm{C}$ for $1 \mathrm{~h}$, and the incubated scaffolds were injected subcutaneously in either back of BALB/c nude mice ( 5 weeks old, $n=4$ per group). This experiment was approved by the Institutional Animal Care and Use Committee of Peking University Health Science Center (LA2014233). Animal experiments were carried out according to the Guidelines of Department of Laboratory Animals (Central South University, China).

\section{In vivo bone formation analyses}

After 8 weeks of osteogenic differentiation in vivo, all nude mice were sacrificed, and the Bio-Oss Collagen was stripped from the subcutaneous skin and fixed in 4\% PFA. The specimens were analyzed using Quantum GX microCT Imaging System (PerkinElmer, CA, USA). BV/ TV and BMD were measured using Quantum Supporting Xcapture Software (PerkinElmer, CA, USA). Then the specimens were decalcified in 10\% EDTA solution for 14 days, followed by embedding with paraffin and sectioning. The sections $(4.0 \mu \mathrm{m})$ were stained with $\mathrm{H}$ and $\mathrm{E}$ (Boster, USA) and Masson Trichrome (Solarbio, Beijing, China). In addition, IHC staining was performed with anti-OCN and anti-OSX to evaluate the expression of osteogenic markers. The images were taken using CKX41 Optical microscope (Olympus, Japan).

\section{Statistical analysis}

All data are presented as Mean \pm SD of three independent experiments and statistical analysis was performed using SPSS Statistics V22.0 (IBM Corporation, Armonk, NY, USA). Two-group data were analyzed using Student's $t$-test, and three-group or multiple-group data were analyzed using one-way ANOVA. A two-tailed $p$-value $<0.05$ was regarded as statistically significant. 


\section{Acknowledgements}

This study was granted by the National Natural Science Foundation of China (No. 51935014, 51905553, 81871494, 81871498, 51705540, 81572577), the Program of Introducing Talents of Discipline to Universities (No.111-2-12), the Hunan Province Natural Science Foundation of China (No.2016JJ1027), JiangXi Provincial Natural Science Foundation of China (20192ACB20005), Guangdong Province Higher Vocational Colleges \& Schools Pearl River Scholar Funded Scheme (2018), the Natural Science Foundation of Guangdong Province (No. 2016A030313010), the Open-End Fund for the Valuable and Precision Instruments of Central South University.

\section{Author details}

${ }^{1} \mathrm{NHC}$ Key Laboratory of Carcinogenesis, Hunan Provincial Tumor Hospital, Central South University, Changsha 410013, China. ${ }^{2}$ The Key Laboratory of Carcinogenesis and Cancer Invasion of the Chinese Ministry of Education, Cancer Research Institute and School of Basic Medical Sciences, Central South University, Changsha 410078, China. ${ }^{3}$ Hunan Key Laboratory of Non-resolving Inflammation and Cancer, Disease Genome Research Center, the Third Xiangya Hospital, Central South University, Changsha 410013, China. ${ }^{4}$ Department of Obstetrics and Gynecology, General Hospital, Shenzhen University, Shenzhen 518053, China. ${ }^{5}$ Jiangxi University of Science and Technology, Ganzhou 341000, China. ${ }^{6}$ State Key Laboratory of High Performance Complex Manufacturing, Central South University, Changsha 410083, China

\section{Conflict of interest}

The authors declare that they have no conflict of interest.

\section{Publisher's note}

Springer Nature remains neutral with regard to jurisdictional claims in published maps and institutional affiliations.

Supplementary Information accompanies this paper at (https://doi.org/ 10.1038/541419-019-2148-2).

Received: 30 September 2019 Revised: 1 November 2019 Accepted: 11 November 2019

Published online: 11 December 2019

\section{References}

1. Shuai, C. J. et al. Antibacterial polymer scaffold based on mesoporous bioactive glass loaded with in situ grown silver. Chem. Eng. J. 374, 304-315 (2019).

2. Shuai, C. J., Wang, B., Yang, Y. W., Peng, S. P. \& Gao, C. D. 3D honeycomb nanostructure-encapsulated magnesium alloys with superior corrosion resistance and mechanical properties. Compos. Part B Eng. 162, 611-620 (2019).

3. Shuai, C. J. et al. Surface modification of nanodiamond: toward the dispersion of reinforced phase in poly-L-lactic acid scaffolds. Int. J. Biol. Macromol. 126, 1116-1124 (2019).

4. Shuai, C. J. et al. nMgO-incorporated PLLA bone scaffolds: enhanced crystallinity and neutralized acidic products. Mater. Design 174, 107801 (2019).

5. Gao, C. et al. Highly biodegradable and bioactive Fe-Pd-bredigite biocomposites prepared by selective laser melting. J. Adv. Res. 20, 91-104 (2019).

6. Gao, C. D., Feng, P., Peng, S. P. \& Shuai, C. J. Carbon nanotube, graphene and boron nitride nanotube reinforced bioactive ceramics for bone repair. Acta. Biomater. 61, 1-20 (2017).

7. Yang, Y. W., Guo, X. N., He, C. X., Gao, C. D. \& Shuai, C. J. Regulating degradation behavior by incorporating mesoporous silica for $\mathrm{Mg}$ bone implants. Acs Biomater Sci Eng 4, 1046-1054 (2018).

8. Haugh, M. G. \& Heilshorn, S. C. Integrating concepts of material mechanics, ligand chemistry, dimensionality and degradation to control differentiation of mesenchymal stem cells. Curr. Opin. Solid State Mater. Sci. 20, 171-179 (2016).

9. Lin, H., Sohn, J., Shen, H., Langhans, M. T. \& Tuan, R. S. Bone marrow mesenchymal stem cells: Aging and tissue engineering applications to enhance bone healing. Biomaterials 203, 96-110 (2018).

10. Liu, Y., Luo, D. \& Wang, T. Hierarchical structures of bone and bioinspired bone tissue engineering. Small 12, 4611-4632 (2016).
11. Diao, Y., Ma, Q., Cui, F. \& Zhong, Y. Human umbilical cord mesenchymal stem cells: osteogenesis in vivo as seed cells for bone tissue engineering. J. Biomed. Mater. Res. A 91, 123-131 (2009).

12. Arutyunyan, I., Elchaninov, A., Makarov, A. \& Fatkhudinov, T. Umbilical cord as prospective source for mesenchymal stem cell-based therapy. Stem Cells Int 2016, 6901286 (2016).

13. Marupanthorn, K., Tantrawatpan, C., Tantikanlayaporn, D., Kheolamai, P. \& Manochantr, S. The effects of TNF-alpha on osteogenic differentiation of umbilical cord derived mesenchymal stem cells. J. Med. Assoc. Thai $\mathbf{9 8}$ (Suppl 3), S34-S40 (2015).

14. Peng, S. et al. An overview of long noncoding RNAs involved in bone regeneration from mesenchymal stem cells. Stem Cells Int 2018, 8273648 (2018).

15. Jia, Q., Jiang, W. \& Ni, L. Down-regulated non-coding RNA (IncRNA-ANCR) promotes osteogenic differentiation of periodontal ligament stem cells. Arch. Oral. Biol. 60, 234-241 (2015)

16. Gao, X., Ge, J., Li, W., Zhou, W. \& Xu, L. LncRNA KCNQ1OT1 promotes osteogenic differentiation to relieve osteolysis via Wnt/beta-catenin activation. Cell Biosci. 8, 19 (2018)

17. Zhuang, W. et al. Upregulation of IncRNA MEG3 promotes osteogenic differentiation of mesenchymal stem cells from multiple myeloma patients by targeting BMP4 transcription. Stem Cells 33, 1985-1997 (2015).

18. Wang, Q. et al. LncRNA MEG3 inhibited osteogenic differentiation of bone marrow mesenchymal stem cells from postmenopausal osteoporosis by targeting miR-133a-3p. Biomed. Pharmacother. 89, 1178-1186 (2017).

19. Gong, Z. M., Tang, Z. Y. \& Sun, X. L. LncRNA PRNCR1 regulates osteogenic differentiation in osteolysis after hip replacement by targeting miR-211-5p. Gene 673, 251-261 (2018).

20. Jin, C. et al. Inhibition of IncRNA MIR31HG promotes osteogenic differentiation of human adipose-derived stem cells. Stem Cells 34, 2707-2720 (2016).

21. Kim, J. et al. RAD6-Mediated transcription-coupled H2B ubiquitylation directly stimulates H3K4 methylation in human cells. Cell 137, 459-471 (2009).

22. Vethantham, $\mathrm{V}$. et al. Dynamic loss of $\mathrm{H} 2 \mathrm{~B}$ ubiquitylation without corresponding changes in H3K4 trimethylation during myogenic differentiation. Mol. Cell Biol. 32, 1044-1055 (2012).

23. Beermann, J., Piccoli, M. T., Viereck, J. \& Thum, T. Non-coding RNAs in development and disease: background, mechanisms, and therapeutic approaches. Physiol. Rev. 96, 1297-1325 (2016).

24. $\mathrm{Xu}$, Z. et al. $\mathrm{H} 2 \mathrm{~B}$ ubiquitination regulates meiotic recombination by promoting chromatin relaxation. Nucleic Acids Res. 44, 9681-9697 (2016).

25. Zhou, V. W., Goren, A. \& Bernstein, B. E. Charting histone modifications and the functional organization of mammalian genomes. Nat. Rev. Genet. 12, 7-18 (2011).

26. Baumann, $U$. et al. Disruption of the PRKCD-FBXO25-HAX-1 axis attenuates the apoptotic response and drives lymphomagenesis. Nat. Med. 20, 1401-1409 (2014).

27. McGourty, C. A. et al. Regulation of the CUL3 ubiquitin ligase by a calciumdependent co-adaptor. Cell 167, 525-538 e514 (2016).

28. Hong, E. J., Villen, J., Gerace, E. L., Gygi, S. P. \& Moazed, D. A cullin E3 ubiquitin ligase complex associates with Rik1 and the Clr4 histone H3-K9 methyltransferase and is required for RNAi-mediated heterochromatin formation. RNA Biol. 2, 106-111 (2005).

29. Thakar, A., Panvin, J. D. \& Zlatanova, J. BRCA1/BARD1 E3 ubiquitin ligase can modify histones $\mathrm{H} 2 \mathrm{~A}$ and $\mathrm{H} 2 \mathrm{~B}$ in the nucleosome particle. J. Biomol. Struct. Dyn. 27, 399-405 (2010).

30. Sinha, K. M., Yasuda, H., Coombes, M. M., Dent, S. Y. \& de Crombrugghe, B. Regulation of the osteoblast-specific transcription factor Osterix by NO66, a Jumonji family histone demethylase. EMBO J. 29, 68-79 (2010).

31. Liu, T. M. \& Lee, E. H. Transcriptional regulatory cascades in Runx2-dependent bone development. Tissue Eng. Part B Rev. 19, 254-263 (2013).

32. Sinha, K. M. \& Zhou, X. Genetic and molecular control of osterix in skeletal formation. J. Cell Biochem. 114, 975-984 (2013).

33. Franceschi, R. T., Ge, C., Xiao, G., Roca, H. \& Jiang, D. Transcriptional regulation of osteoblasts. Ann. N.Y. Acad. Sci. 1116, 196-207 (2007).

34. Komori, T. Runx2, an inducer of osteoblast and chondrocyte differentiation. Histochem. Cell Biol. 149, 313-323 (2018).

35. Nishio, Y. et al. Runx2-mediated regulation of the zinc finger Osterix/Sp7 gene. Gene 372, 62-70 (2006).

36. Manfiolli, A. O. et al. FBXO25-associated nuclear domains: a novel subnuclear structure. Mol. Biol. Cell 19, 1848-1861 (2008). 
37. Jang, J. W. et al. A novel Fbxo25 acts as an E3 ligase for destructing cardiac specific transcription factors. Biochem. Biophys. Res. Commun. 410, 183-188 (2011).

38. Teixeira, F. R. et al. FBXO25 regulates MAPK signaling pathway through inhibition of ERK1/2 phosphorylation. Arch. Biochem. Biophys. 621, 38-45 (2017).

39. Sepulveda, $\mathrm{H}$. et al. Epigenetic signatures at the RUNX2-P1 and Sp7 gene promoters control osteogenic lineage commitment of umbilical cord-derived mesenchymal stem cells. J. Cell Physiol. 232, 2519-2527 (2017).

40. Baarends, W. M. et al. Silencing of unpaired chromatin and histone H2A ubiquitination in mammalian meiosis. Mol. Cell Biol. 25, 1041-1053 (2005).

41. Muller, J. \& Verrijzer, P. Biochemical mechanisms of gene regulation by polycomb group protein complexes. Curr. Opin. Genet Dev. 19, 150-158 (2009).

42. $\mathrm{Hu}, \mathrm{H}$. et al. CRL4B catalyzes H2AK119 monoubiquitination and coordinates with PRC2 to promote tumorigenesis. Cancer Cell 22, 781-795 (2012).

43. Henry, K. W. et al. Transcriptional activation via sequential histone H2B ubiquitylation and deubiquitylation, mediated by SAGA-associated Ubp8. Genes Dev. 17, 2648-2663 (2003).

44. Woo Park, J. et al. RE-IIBP Methylates H3K79 and Induces MEIS1-mediated Apoptosis via H2BK120 Ubiquitination by RNF20. Sci. Rep. 5, 12485 (2015).

45. Strikoudis, A., Lazaris, C., Ntziachristos, P., Tsirigos, A. \& Aifantis, I. Opposing functions of $\mathrm{H} 2 \mathrm{BK} 120$ ubiquitylation and $\mathrm{H} 3 \mathrm{~K} 79$ methylation in the regulation of pluripotency by the Paf1 complex. Cell Cycle 16, 2315-2322 (2017).

46. Chen, S., Li, J., Wang, D. L. \& Sun, F. L. Histone H2B lysine 120 monoubiquitination is required for embryonic stem cell differentiation. Cell Res. 22, 1402-1405 (2012).
47. Fuchs, G. et al. RNF20 and USP44 regulate stem cell differentiation by modulating H2B monoubiquitylation. Mol. Cell 46, 662-673 (2012).

48. Sheen-Chen, S. M. et al. Epigenetic histone methylation regulates transforming growth factor beta-1 expression following bile duct ligation in rats. J. Gastroenterol. 49, 1285-1297 (2014).

49. Zhou, $\mathrm{X}$. et al. Hypoxia induces trimethylated $\mathrm{H} 3$ lysine 4 by inhibition of JARID1A demethylase. Cancer Res. 70, 4214-4221 (2010).

50. Kim, J. et al. The $\mathrm{n}$-SET domain of Set1 regulates $\mathrm{H} 2 \mathrm{~B}$ ubiquitylationdependent H3K4 methylation. Mol. Cell 49, 1121-1133 (2013).

51. Wang, C. et al. LncRNA structural characteristics in epigenetic regulation. Int. J. Mol. Sci. 18, 2659 (2017).

52. Shuai, C. J. et al. A combined nanostructure constructed by graphene and boron nitride nanotubes reinforces ceramic scaffolds. Chem Eng J 313, 487-497 (2017)

53. Gao, C. D., Yao, M., Shuai, C. J., Peng, S. P. \& Deng, Y. W. Nano-SiC reinforced Zn biocomposites prepared via laser melting: Microstructure, mechanical properties and biodegradability. J Mater Sci Technol 35, 2608-2617 (2019).

54. He, S. et al. HMG-box transcription factor 1: a positive regulator of the G1/S transition through the Cyclin-CDK-CDKI molecular network in nasopharyngeal carcinoma. Cell Death Dis. 9, 100 (2018).

55. Schneider Gasser, E. M. et al. Immunofluorescence in brain sections: simultaneous detection of presynaptic and postsynaptic proteins in identified neurons. Nat. Protoc. 1, 1887-1897 (2006).

56. Zhong, Y. et al. The interaction of Lin28A/Rho associated coiled-coil containing protein kinase2 accelerates the malignancy of ovarian cancer. Oncogene $\mathbf{3 8}$, 1381-1397 (2019). 